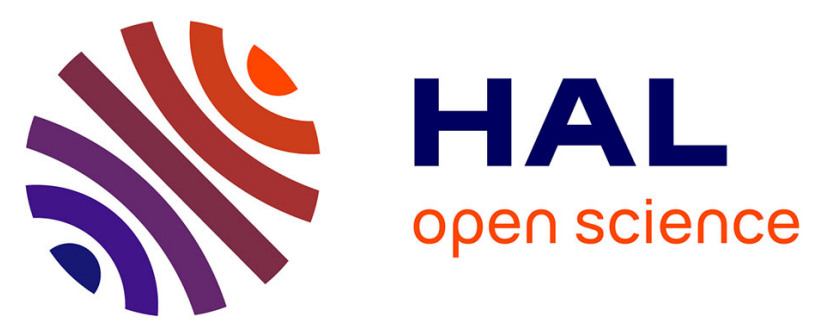

\title{
Surveiller en éradiquant : l'importance des " médiateurs de la surveillance" et des réseaux informels dans la surveillance des risques sanitaires et environnementaux
}

Giovanni Prete

\section{- To cite this version:}

Giovanni Prete. Surveiller en éradiquant: l'importance des "médiateurs de la surveillance " et des réseaux informels dans la surveillance des risques sanitaires et environnementaux. Sociologie du Travail, 2008, 50 (4), pp.489-504. 10.1016/j.soctra.2008.09.002 . halshs-01236020

\section{HAL Id: halshs-01236020 \\ https://shs.hal.science/halshs-01236020}

Submitted on 1 Dec 2015

HAL is a multi-disciplinary open access archive for the deposit and dissemination of scientific research documents, whether they are published or not. The documents may come from teaching and research institutions in France or abroad, or from public or private research centers.
L'archive ouverte pluridisciplinaire HAL, est destinée au dépôt et à la diffusion de documents scientifiques de niveau recherche, publiés ou non, émanant des établissements d'enseignement et de recherche français ou étrangers, des laboratoires publics ou privés. 


\title{
Titre : Surveiller en éradiquant: l’importance des «médiateurs de la surveillance» et des réseaux informels dans la surveillance des risques sanitaires et environnementaux
}

\author{
Auteur : Giovanni Prete
}

In Sociologie du Travail, Vol 50 - N 4, pp. 489-504, 2008

\begin{abstract}
Résumé
Les dispositifs de surveillance sont devenus un des principaux modes d'action publique vis-àvis des risques sanitaires et environnementaux. Leur fonctionnement repose souvent sur la coopération entre des acteurs aux intérêts contradictoires. Cet article analyse les modalités de cette coopération lorsque ni la coercition, ni les incitations ne suffisent à l'assurer. L'étude du suivi d'un virus attaquant des cultures agricoles met en évidence l'existence d'arrangements par rapport aux cadres formels de la surveillance. Ces arrangements, qui rendent possible la circulation de l'information sanitaire, sont favorisés par l'activité d'acteurs — qualifiés de « médiateurs de la surveillance » - qui, grâce à un ancrage local durable et des compétences techniques, articulent différents mondes sociaux. L'article montre ainsi la nécessité de prendre en compte la nature politique des données recueillies au cours des activités de surveillance et d'étudier en détail les traductions multiples dont elles font l'objet et qui sont les conditions de leur recueil.
\end{abstract}

Mots clés : Surveillance ; Risque ; Agriculture ; Marginal-sécant ; Médiateur ; Pathogène des plantes ; Biosécurité ; France

\begin{abstract}
Surveillance measures, now a major form of public action in relation to health and environmental risks, often rely on cooperation among parties with contradictory interests. This cooperation is analyzed in cases when neither coercion nor incentives suffice to obtain it. A study of an attack by a virus on crops has brought to light arrangements that contrast with the formal setup for surveillance. These arrangements made it possible for health-related information to circulate. They were facilitated by the activity of "surveillance mediators" who were able - thanks to their durable local roots and their technical qualifications - to relate to different social worlds. Light is thus shed on the need both to take into account the political nature of the data collected during surveillance activities and to examine the many forms they take — forms that are the very conditions for data collection.
\end{abstract}

Keywords : Surveillance, Health and environmental risks, Agriculture, Cross-cutting ties, Mediator, Plant pathogens, Biosecurity, France 
La surveillance s'est imposée depuis quelques années comme une activité clé de la sécurité sanitaire et environnementale. Dans le domaine alimentaire, la surveillance est un élément central du dispositif prôné par la Commission européenne (cf. Livre blanc sur la sécurité alimentaire, de 1999) suite aux différentes crises des années 1990. Dans les domaines animal et végétal, la mise sur agenda des maladies invasives ou émergentes ${ }^{1}$ s'est également traduite par une multiplication de discours normatifs sur la gestion des problèmes sanitaires et environnementaux qui mettent l'accent sur la nécessité de renforcer les activités de surveillance ${ }^{2}$ Devenue un des modes principaux d'action publique vis-à-vis des risques sanitaires et environnementaux, la surveillance vise ainsi à tracer, au-delà de l'humain, toutes les espèces vivantes (Lascoumes, 1993) et les choses (Torny, 1998) au travers un ensemble d'activités diverses: collecte de données sur le terrain, système de « vigilance » et d' « alerte », activités de contrôle officiel (Demortain, 2006).

Les autorités sanitaires ont rarement la capacité de réaliser ces différentes activités seules et ont besoin de coopérer avec d'autres acteurs dont les intérêts par rapport à l'objectif de surveillance peuvent être contradictoires. L’obligation de coopération est généralement inscrite dans le droit mais les exemples historiques sont nombreux qui attestent des limites des dispositifs formels et coercitifs de surveillance sanitaires ${ }^{3}$. La surveillance peut en effet créer une situation de « dilemme social » (Kollock, 1998), dans laquelle la rationalité de décisions d'acteurs individuels entraîne une diminution du bien-être collectif — ne pas coopérer au dispositif de surveillance en ne notifiant pas la contamination de sa production, par exemple, amène le développement d'une maladie dans une zone de production. Une issue consiste alors à jouer sur les préférences des acteurs, notamment en les incitant financièrement (indemnisations) à coopérer. Cependant, cette solution n'est ni toujours opérante, ni toujours disponible.

À partir d'une étude de cas dans le domaine de la santé végétale, cet article analyse les modalités d'organisation des dispositifs de surveillance quand ni le droit, ni des dispositifs d'incitation ne sont suffisants pour assurer la coopération nécessaire à leur fonctionnement. En s'inscrivant dans la continuité d'auteurs qui ont insisté sur l'importance d'appréhender les dispositifs de collecte de données dans leur fonctionnement concret (Douglas, 1967), il montre que les difficultés de la coopération autour de dispositifs formalisés de surveillance n'empêchent pas la circulation de l'information sanitaire dans les systèmes d'acteurs concrets (Crozier et Friedberg, 1977) de la surveillance ${ }^{4}$.

Plus précisément, cet article met en lumière pourquoi et comment des arrangements par rapport aux cadres formels de la surveillance se développent et permettent la coopération d'acteurs appartenant à différents mondes sociaux. Il permet, en discutant la littérature sociologique sur les intermédiaires, de qualifier certains acteurs de «médiateurs

\footnotetext{
${ }^{1}$ Nous ne discuterons pas dans cet article de ces concepts, de leur pertinence et de leur utilisation, qui ont pu faire l'objet d'un regard critique. Pour une tentative de conceptualisation, voir Grmek (1993).

${ }^{2}$ Cette tendance est très claire dans le domaine de la santé animale. Dans le domaine végétal, généralement moins connu, il existe aujourd'hui des normes internationales (CIPV) et un ensemble de projets internationaux (par exemple, les projets « Daisie ", " Invasive Species », ou le programme " Biovigilance » du ministère de l’Agriculture franc,aise (Delos et al., 2005) qui visent à encadrer les activités de surveillance des maladies émergentes, que celles-ci soient qualifiées d'espèces invasives, exotiques ou nuisibles.

${ }^{3}$ A. Woods signale, par exemple, qu'en 1922 comme en 2001, de nombreux éleveurs cachèrent les cas de foot and mouth disease aux autorités sanitaires du Royaume-Uni (Woods, 2004).

${ }^{4}$ Dans le domaine sanitaire, et plus particulièrement dans les domaines de la santé humaine et de la santé animale, il existe plusieurs tentatives définitionnelles et typologiques de la surveillance : voir par exemple Dufour et La Vieille (2000) ; Thurmond (2003). Dans cet article, nous emploierons indifféremment les termes de surveillance et d'épidémiosurveillance pour décrire un ensemble assez large d'activités à savoir " toute activité de collecte et de compilation de données sur la présence et le développement d'un pathogène dans une zone géographique délimitée ». Cette définition positive ne dénie pas la pertinence, quand il s’agit d’interroger la nature d'une institution ou d'une activité, de distinguer par exemple la veille sanitaire, de la surveillance ou de la police (Buton, 2006).
} 
de la surveillance » qui favorisent la coopération en articulant différents mondes sociaux, dans lesquels ils ont su construire des relations de confiance et mobiliser dans le temps des compétences techniques et une capacité d'empathie. Cette analyse amène en conclusion à discuter les tentatives de mise en place de dispositifs de surveillance qui ne prennent pas en compte la dimension politique des données sanitaires et qui ne s’interrogent pas sur les conditions concrètes du succès de leur recueil ${ }^{5}$.

\section{La difficile mise en dispositif de l’épidémiosurveillance}

\subsection{L'arrivée d'un problème phytosanitaire porteur d'incertitude}

Cette section examine la difficulté à faire collaborer les acteurs concernés ${ }^{6}$ dans des dispositifs de surveillance visant à suivre le développement d'un virus qui attaque principalement les cultures de tomate. Par souci de clarté, nous allons présenter rapidement ces acteurs avant de rentrer dans le détail de leurs interactions. Nous les regroupons en trois grandes catégories - administration, profession, recherche — qui sont utilisées de manière récurrente par les acteurs à la fois au cours des entretiens réalisés et dans le cadre des interactions entre eux. Mobiliser ces catégories ne signifie pas qu'elles soient complètement adéquates pour définir les phénomènes qui nous intéressent. Au contraire, la démonstration visera à souligner l'importance d'acteurs qui circulent entre les mondes sociaux décrits par ces catégories ${ }^{6}$.

\section{- $\quad$ Les acteurs de l'administration}

Il s’agit ici du service de la protection des végétaux (PV), qui dépend du ministère de l'Agriculture. Il est chargé d'appliquer les normes internationales (CIPV), européennes (directive 2000/29/CE) et nationales relatives à la santé des végétaux. Il s’appuie sur les agents des services régionaux de la protection des végétaux (SRPV) pour mener son action et, plus particulièrement dans le cas étudié, sur une antenne locale du SRPV située au cœur de la zone de production concernée.

\section{- $\quad$ Les acteurs de la profession}

La «profession » est une catégorie particulièrement large. Elle regroupe : des animateurs des deux syndicats professionnels les plus implantés chez les maraîchers dans la région ; des représentants des organisations de producteurs (OP). Ces organisations représentent les producteurs dans des comités économiques officiels et gèrent des aides financières allouées aux producteurs; des techniciens qui appuient les maraîchers dans la conduite de leurs serres et qui dépendent: soit de la chambre d'agriculture, une organisation consulaire qui a vocation à apporter un soutien technique et administratif à tous les producteurs; soit de la FRUITO, une station professionnelle locale financée en grande partie par la chambre d'agriculture; soit d'un groupe de développement agricole (GDA), c'est-à-dire un ensemble d'agriculteurs regroupés pour collectiviser le financement d'un technicien; soit des organisations de producteurs (OP).\$

\footnotetext{
${ }^{5}$ Cet article est basé sur un travail d’enquête approfondi mené depuis 2004 en utilisant des techniques variées. Nous avons rencontré plus de 70 personnes dans le cadre d'entretiens semi-directifs, de manière parfois répétée. Nous avons également pu assister à des réunions regroupant plusieurs des acteurs interrogés. Enfin, nous avons collecté, à chaque fois que cela était possible, des documents d'archives permettant de tracer l'activité de ces personnes depuis la fin des années 1990.

${ }^{6}$ Par acteurs concernés, nous nous référons ici aux acteurs qui se sont révélés actifs dans les systèmes d'acteurs concrets mis en évidence au cours de l'enquête. Il serait évidemment intéressant de s’interroger sur les raisons de l'absence de certains acteurs dans ces systèmes alors que leur participation pourrait être attendue. Cette réflexion dépasserait cependant l’objectif de cet article.
} 
Il s'agit ici essentiellement d'agents de l'institut national de la recherche agronomique (INRA). Deux équipes de l'INRA sont plus particulièrement mobilisées dans la situation étudiée. La première est un groupe de chercheurs - l’équipe Fractale - spécialistes de « lutte biologique ». Ces chercheurs collaborent avec un deuxième groupe constitué d’ingénieurs agronomes basés dans une station expérimentale en maraîchage située au cœur de la zone de développement du virus.

Ces acteurs sont confrontés à une épidémie de Tomato Yellow Leaf Curl Virus (TYLCV), déclarée officiellement par le service de la PV en 2003 dans un département français. Dangereux virus pour les plantes de la famille des solanées et plus particulièrement les cultures de tomate, le virus est classé organisme de quarantaine au niveau de l'Union européenne ${ }^{7}$, de même que son vecteur, l'aleurode Bemisia tabaci ${ }^{8}$ La présence de ce virus dans d'autres pays européens depuis la fin des années 1990, puis la découverte d'un cas isolé en 1999 dans un autre département français, enfin le développement de B. tabaci en France, à partir de 2000, avaient alerté sur la possibilité d'un évènement comme l'épidémie de 2003. Cette année là, la PV réalise une prospection systématique des exploitations : dès le mois de juillet, ce sont plus du tiers des producteurs de tomate sous serres qui sont touchés par le virus?

Plusieurs incertitudes accompagnent ce constat : le virus est-il présent durablement dans la zone de production ? Si non, sa présence exceptionnelle est-elle liée à une introduction accidentelle, par l'importation de plants contaminés, ou à la chaleur de l'été caniculaire qui a favorisé le développement du vecteur du virus ? La résolution de ces incertitudes passe par la mise en place d'un dispositif d'épidémiosurveillance qui permettrait d'évaluer la progression de la maladie, son maintien pendant l'hiver, ou encore de collecter des échantillons d'insecte et de virus pour tenter, aux moyens d'outils génétiques, d'en retracer l'origine. Pourtant, le cadre réglementaire mis en place pour faire face au problème $T Y L C V$ rend très difficile la mise en place d'un tel dispositif et plus généralement la coopération entre l'administration, la profession et la recherche.

\subsection{Difficile coopération autour de l'objectif de surveillance}

En 2000 et 2002, les autorités sanitaires ont rédigé des mesures drastiques (arrêtés de lutte obligatoire) visant à l'éradication du problème phytosanitaire, qui incitent les agriculteurs à dissimuler l'apparition du virus dans leurs serres. Les mesures impliquent que toute parcelle de culture que l'on découvre contaminée par le TYLCV à un taux supérieur à un plant pour mille soit arrachée. Elles ne s’accompagnent pas de garanties d'indemnisations. Aussi, les producteurs ne perçoivent-ils aucun intérêt à transmettre l'information sur l'état sanitaire de leurs serres aux services administratifs. En effet, révéler l'existence d'un plant malade les expose à un risque financier important compromettant notamment leur capacité à amortir les investissements considérables que représentent les serres ${ }^{10}$. Les producteurs préfèrent donc garder secret l'état sanitaire de leurs cultures et

\footnotetext{
${ }^{7}$ À ce titre, les plants de tomate qui entrent en France doivent être accompagnés d'un passeport phytosanitaire qui atteste de leur propreté au regard du virus et de l'insecte.

${ }^{8} \mathrm{~B}$. tabaci est un aleurode ou mouche blanche mesurant de 1 à $2 \mathrm{~mm}$ de long à l'âge adulte. Polyphage, il se nourrit en piquant et en suc,ant la plante hôte sur laquelle il se trouve. Plusieurs espèces d'aleurodes existent, Trialeurodes vaporariorum et B. tabaci sont présents en France. B. tabaci occasionne des dégâts directs qui fragilisent les plantes. Il est porteur de nombreux virus dont le plus dangereux est le TYLCV. Ce virus provoque un jaunissement et/ou un enroulement des feuilles de tomate. Infectée, la plante voit son développement bloqué et ne produit plus de fruits.

${ }^{9}$ La France produit alors 550000 tonnes de tomate en frais par an, plus de 80 \% sous serres. Dans la zone concernée, ilya environ 150 producteurs de tomate sous serre en 2003.

${ }^{10}$ De manière plus indirecte, un producteur qui révèle que sa serre est contaminée court le risque d'être accusé par d'autres producteurs d'être responsable de l'infestation de leurs serres. Cela est arrivé à l'automne 2004 dans
} 
interdisent aux techniciens qui y ont accès d'en faire état à des tiers. S’ils découvrent du TYLCV dans leurs serres, ils enlèvent les plants contaminés un par un, espérant pouvoir contenir la progression de la maladie et continuer à vendre la production en cours. Ainsi, bien que les textes de lois soulignent que toute découverte du TYLCV doit être notifiée aux services officiels sous peine d'amendes lourdes et de prison, la PV doit elle-même faire des prospections dans les serres pour obtenir de l'information sur l'état réel de la situation sanitaire. Ayant des moyens matériels et humains limités pour réaliser ces prospections, l'administration estime ne pas avoir une vision adéquate de la présence et de la progression du virus.

Cette difficulté est soulevée à plusieurs reprises dans des rencontres qui réunissent les représentants des pouvoirs publics et les représentants syndicaux des producteurs: les premiers conditionnant une discussion sur des indemnisations en cas d'arrachage à une transparence sur l'état sanitaire des serres ; les seconds conditionnant la remontée de l'information à des garanties d'indemnisation. Si le cadre réglementaire rend difficile la mise en place d'un dispositif d'épidémiosurveillance animé par l'administration de la PV, il rend également problématique tout dispositif de suivi épidémiologique qui serait mis en place dans le cadre de programmes de recherche.

Pour les acteurs de la recherche, mener des activités scientifiques sur les organismes de quarantaine implique des contraintes particulières : les expérimentations doivent être menées dans des espaces confinés agréés ; la circulation des échantillons est soumise à l'obtention d'autorisations délivrées par l'administration; enfin les chercheurs sont dans l'obligation de déclarer aux autorités sanitaires toute détection du pathogène qu'ils pourraient faire dans les échantillons qu'ils manipulent et les cultures qu'ils visitent. Ce dernier point est particulièrement intéressant pour notre propos. En effet, en 2004, des chercheurs de l'équipe Fractale de l'INRA, qui est implantée hors de la zone de production, réfléchissent à la mise en place d'un programme de suivi épidémiologique. Pour réaliser ce travail, le principal problème de l'équipe est l'accès aux serres dans lesquelles les échantillons pourraient être collectés.

«Si tu veux pour résumer en deux mots, en France, on a des symptômes de TYLCV et on arrache pratiquement la serre et on n'a pas de dédommagements financiers contrairement à beaucoup d'autres calamités agricoles. . ça veut dire qu'on est en pleine opacité avec la production, qu'on a des difficultés à aller dans les zones de production, que si, par exemple [un collègue chercheur] trouve autour des serres du TYLCV, il va être obligé de le déclarer séance tenante dès qu'il a vu qu'il s'agit bien de TYLCV, et ça signifie qu'il y a des mesures particulières de surveillance qui sont prises par la PV dans le canton, et ça veut dire que le prochain coup les agriculteurs ils vont t'attendre avec le 22 long rifle. » (chercheur Fractale).

Pour eux, la question qui se pose est de savoir comment obtenir l'accès à des exploitations pour produire des données qui pourraient obliger ces mêmes agriculteurs à devoir arracher leurs cultures de tomate.

Étant donné le cadre réglementaire, nous avons montré qu'il était, pour les producteurs, hors de question de participer à tout dispositif de surveillance dont la finalité ou le fonctionnement impliquerait la remontée d'information vers les pouvoirs publics. À l'inverse, leurs représentants mettent en place, dès 2002, un dispositif de contrôle de la circulation de cette information: une «cellule de veille professionnelle».

\subsection{Une surveillance "professionnelle» confinée}


Cette « cellule de veille professionnelle » est animée par les principaux représentants syndicaux des maraîchers. Elle réunit, avant tout, les techniciens, ainsi que des représentants des organisations de producteurs (OP) de la zone de production. Cette cellule se réunit plusieurs fois par an, non seulement pour réfléchir aux meilleurs moyens de lutte contre $B$. tabaci et ses virus, mais également pour organiser le contrôle de la circulation des données sur la situation sanitaire, vis-à-vis des médias et vis-à-vis des pouvoirs publics.

Cette cellule est la formalisation d'un réseau informel d'échange constitué des techniciens et des principaux représentants politiques agricoles. Ces acteurs ont l'habitude d'échanger dans des groupes de travail ou des réunions sur des thématiques autres que les virus de B.tabaci. Certains techniciens ont notamment, au cours de leur carrière, été amenés à travailler dans des organisations communes. Ils ont développé au cours de ces échanges des relations de confiance ${ }^{11}$ qui leur permettent, malgré la réticence des producteurs, d'échanger entre eux les informations sur le virus qu'ils collectent au cours de leurs activités d'appui technique dans les serres. Ces échanges sont régulés à la fois par la nature des relations interpersonnelles entre chacun des techniciens et par certaines règles tacites communément partagées qui impliquent, principalement, d'échanger oralement et de ne jamais mentionner le lieu exact et/ou le nom du propriétaire de la serre concernée.

Dans ce réseau technique informel, le responsable technique de la FRUITO joue un rôle particulier. La FRUITO réalise depuis plusieurs années des essais (sur les variétés, les techniques de gestion des cultures) très appliqués dans les serres et parcelles de certains maraîchers, dont elle diffuse les résultats à l'ensemble des producteurs de la zone. Cette activité donne l'opportunité à son responsable technique d'être régulièrement en contact avec de nombreux producteurs et tous les techniciens du département. Il profite de ces échanges pour compiler l'information sur l'évolution de la situation sanitaire, ce qui lui permet d'élaborer des cartes qualitatives qui, bien qu'imprécises, semblent constituer d'assez justes représentations de l'évolution de la présence du TYLCV. Sous le contrôle des représentants syndicaux, il fait circuler ces informations et ces cartes, modulant son discours en fonction du public visé.

"Au niveau de Bemisia il y a des trucs qu'on ne peut pas dire, il y a des choses qu'on ne peut pas divulguer. C'est à ce niveau là que les liens personnels interviennent. [...] Disons qu'il y a plusieurs niveaux de communication. Un niveau de communication vers le public où on ne veut pas que se sache que Bemisia est vecteur de virus très dangereux. Si ça se savait ce serait une catastrophe.

(Même s’il n'y a pas de conséquences pour la santé humaine ?). Le public ne fait pas la différence. Après il y a un niveau de communication vers les techniciens de terrain. On va un peu plus loin. Après le discours technique peut nuire au discours commercial. Donc il y a une différence entre ces deux voies de communication.

(Par exemple au niveau de la cellule de veille vous êtes langue de bois ?). Non pas au niveau de la cellule de veille. » (technicien FRUITO).

Pour les acteurs professionnels participer à ce réseau informel d'échange de données

\footnotetext{
${ }^{11}$ Nous adoptons ici la conception de G. Simmel de la confiance telle que définie dans son texte sur le secret et les sociétés secrètes (Simmel, 1999). La confiance est « une hypothèse sur une conduite future, assez sûre pour qu'on fonde sur elle l'action pratique, la confiance est aussi un état intermédiaire entre le savoir et le non-savoir sur autrui. Celui qui sait tout n’a pas besoin de faire confiance, celui qui ne sait rien ne peut raisonnablement même pas faire confiance ». Dans ce cadre, la confiance est très liée au savoir que l'on a sur autrui. Dans ce texte, pour G. Simmel, les relations sociales ne sont possibles que parce que les individus savent des choses les uns des autres : c'est l'image que l'on a de l'autre qui structure nos relations avec lui et ce sont nos relations avec lui qui déterminent nos images (p. 349).
} 
phytosanitaires est primordial. Cela permet aux techniciens de la zone de production d'ajuster les essais qu'ils mettent en place (de variété, de stratégie de traitement phytosanitaire) et les préconisations qu'ils font aux producteurs en fonction de l'évolution de la situation sanitaire. Pour les responsables syndicaux, il est important d'avoir connaissance de l'évolution de la situation sanitaire pour ajuster les exigences formulées dans les négociations avec les pouvoirs publics sur les indemnisations et les aides aux producteurs.

Nous venons de voir comment l'arrivée d'un nouveau virus avait rendu nécessaire la mise en place de dispositifs d'épidémiosurveillance impliquant la coopération d'acteurs hétérogènes pris dans des relations d'interdépendance. L'administration, qui n’a pas les moyens de surveiller toutes les serres de production, a besoin de la coopération des producteurs, techniciens et représentants syndicaux pour connaître l'évolution du virus. Des chercheurs dépendent également de ces mêmes acteurs pour mettre en place un dispositif de suivi épidémiologique. La mise en place d'un mécanisme d’indemnisation intégral pourrait permettre d’obtenir cette coopération mais n'est alors pas envisagée comme une solution « ouverte» dans le cours des négociations.

La littérature sociologique a largement insisté sur le rôle que peuvent jouer les arrangements informels et les relations interindividuelles dans la mise en œuvre de coopérations quand l'autorité de la règle formelle ne suffit pas à l'assurer. Notre étude permet d'interroger les modalités concrètes de ces arrangements et montrer qu'ils reposent en grande partie sur deux acteurs pivots de la surveillance. Dans la partie suivante, nous allons analyser leur action et montrer comment et pourquoi ils s'engagent dans une activité que nous qualifierons de travail de médiation afin de rendre compte à la fois de la nature instable et des motivations complexes qui la sous-tendent. Ainsi, nous montrerons comment ces médiateurs, qui ont construit dans le temps des relations de confiance avec différents mondes sociaux, permettent un dépassement des blocages créés par le cadre formel de la surveillance.

\section{La surveillance « au concret» comme travail de médiation}

\subsection{Deux acteurs pivots de la surveillance « au concret»}

Dans la situation étudiée, deux acteurs jouent un rôle primordial dans l'existence d'arrangements informels et le développement d'une coopération entre les différents mondes sociaux concernés. Ces deux acteurs ont des rôles institutionnels bien différents vis-à-vis du suivi du virus. Le premier est le responsable local d’une antenne déconcentrée du service régional de la protection des végétaux (SRPV). L'alerte et l'investigation sur le pathogène font partie de ses tâches officielles. Le second est une station expérimentale ${ }^{12}$ de l'INRA implantée dans la zone du foyer. La réglementation lui impose de notifier toute découverte de la maladie sur ses parcelles et d'appliquer les mesures de lutte dans le cas échéant. Pourtant, ces deux acteurs peuvent être rapprochés en ce qu'ils facilitent tous deux la surveillance du $T Y L C V$, de deux façons principalement : premièrement, ils relayent de l'information sur la situation sanitaire ; deuxièmement, ils convainquent différents acteurs de la surveillance de la nécessité de prendre en compte le point de vue des autres acteurs.

L'agent du SRPV participe à de multiples réseaux d'information technique et réunions professionnelles. Il a développé avec certains techniciens des relations qui lui permettent

\footnotetext{
${ }^{12}$ Nous avons considéré que la station pouvait être analysée comme un acteur collectif dans la mesure où il y avait, au regard des enjeux de la situation, une forte cohérence des visions et prises de position de ses membres et un accord partagé des décisions prises par son directeur.
} 
d'obtenir, hors du cadre de ses propres prospections officielles, des informations sur l'évolution du virus dans les serres de production. Par exemple, en 2002, c'est un échange avec un technicien de la zone qui lui permet de "découvrir » le premier cas de virus inscrit en lutte obligatoire dans une serre de tomate.

$\mathrm{Au}$ cours de la réalisation des plans de surveillance, il adopte des pratiques " accommodantes » avec le cadre réglementaire. Il lui arrive, par exemple, de faire signer les notifications d'arrachage obligatoire par ses supérieurs au lieu de les signer lui même, ce qui permet de reporter dans le temps la mise en application de cet arrachage et de reporter sur ses supérieurs hiérarchiques la responsabilité de la décision d’arracher. Par ailleurs, comme le décrit l'extrait d'entretien ci-dessous, il adopte lors des visites des serres des méthodes d'observation qui lui donnent la possibilité d’identifier les contaminations importantes mais lui permettent de ne pas forcément mettre en évidence l'existence de «petits » foyers.

"(Vous êtes accommodant avec le seuil d'une plante malade sur mille ?). Je n'irai pas jusque là, mais disons que je n'irai pas fouiller. Il n'est pas question d'aller dans toutes les serres rang par rang, etc. On rentre dans les serres, en général dans l'allée centrale face à l'entrée principale, par laquelle rentre le vecteur... on va pas fouiller partout, ce qu'on voit de l'allée centrale donne une indication, maintenant il pourrait y avoir d'autres foyers avec carreau cass ${ }^{13} e$ mais généralement ce qui est vu de l'allée centrale est un bon indicateur. » (responsable antenne locale PV).

Ainsi, il permet à des producteurs dont les serres sont faiblement infectées d'arracher et de remplacer eux-mêmes leurs plantes au fur et à mesure qu'elles sont contaminées. L'existence de ces "accommodements » dans le travail d'inspection ne renvoie pas ici à un simple détournement de l'ordre juridique mais à une volonté — bien décrite par la littérature sur le travail d'inspection (Dodier, 1991 ; Bonnaud, 2005) — d'inscrire le contrôle dans une relation pédagogique. L'inspection est l'occasion pour l'agent administratif d'informer les producteurs sur la réglementation et de les inciter à adopter des pratiques en matière de gestion des cultures considérées comme nécessaires pour se prémunir du virus. De manière symétrique, l'inspecteur se fait le relais auprès de sa hiérarchie des préoccupations des producteurs et de leur volonté d'obtenir des aides financières et un allégement du plan d'éradication.

Implantée au cœur de la zone de production, la station expérimentale de l’INRA mène depuis une trentaine d'années des expérimentations sur les productions maraîchères sous abri qu'elle élabore avec le souci de répondre aux «besoins professionnels ».

Suite au foyer de 2003, elle facilite la circulation de l'information entre les différents groupes concernés par le virus. Premièrement, elle relaie de l'information entre le monde administratif et le monde productif agricole. En effet, en 2003 la station expérimentale de l'INRA est la première organisation de la zone à déclarer publiquement la présence de TYLCV dans ses serres et à arracher intégralement ses cultures, là où se déroulent des essais expérimentaux. Par la suite, son directeur est invité à participer à plusieurs réunions de la « cellule de veille professionnelle ». Cela lui permet, en plus des contacts qu'il a de manière plus informelle avec des producteurs et des techniciens serristes, d'être tenu au courant de l'évolution de la situation sanitaire. Parallèlement, la station INRA garde des liens privilégiés avec le représentant de la PV. Le directeur de la station tient celui-ci au courant d'informations qu'il collecte au moment où les acteurs professionnels se replient dans le secret.

${ }^{13}$ L'idée exprimée est qu’un carreau cassé dans une serre étant un lieu potentiel d’introduction du vecteur du TYLCV, il est possible que des plantes malades se trouvent dans des allées secondaires, à proximité de vitres brisées. 
Deuxièmement, la station facilite la création d'un dispositif limité de recherche sur l'épidémiologie du pathogène en servant d'intermédiaire entre l'équipe Fractale et des producteurs. En 2006, « en attendant que la réglementation change » (chercheur Fractale), l'équipe de chercheurs met en place un dispositif expérimental afin de collecter des données qualitatives sur la présence du virus dans la zone et de tester des hypothèses sur sa circulation. Concrètement, cinq producteurs acceptent que des cultures sensibles au TYLCV (tomate et autres) soient plantées à proximité de leurs serres de tomate et qu'un suivi épidémiologique soit fait sur ces plantes-hôtes. Comme condition à cette participation, les chercheurs garantissent oralement aux producteurs que s'ils trouvent du TYLCV en extérieur, ils n'avertiront l'administration qu'après la fin de la période de production des plants de tomate. Relativement confidentielle, la participation des producteurs au dispositif expérimental ne donne pas lieu à une rétribution financière du producteur mais s'accompagne d'échanges non monétaires (fourniture de matériel ou de conseils) ${ }^{14}$. Pour mettre en place cette expérimentation, l'intervention du directeur et d'un ingénieur de la station INRA a été primordiale. C'est par leur intermédiaire que les chercheurs de Fractale ont pu identifier les producteurs et les convaincre de s'engager dans un dispositif qui pourrait les exposer à voir leurs cultures arrachées. La station INRA a soit contacté directement des producteurs, soit demandé au technicien de la chambre d'agriculture, avec qui elle a des relations privilégiées, de lui en indiquer qui pourraient être d'accord pour participer à l'expérimentation. Son intermédiation est, pour les professionnels, une garantie que la confidentialité sera respectée.

Comme l'agent de la PV dont nous avons décrit l'action précédemment, la station INRA a également cherché à favoriser un accord sur la gestion de la situation sanitaire entre les différents acteurs concernés.

«Moi, j'intervenais dans les coulisses dans la mesure où je n'avais pas informé les producteurs que j'étais en contact avec la PV et que mon objectif c'était surtout de faciliter cette rencontre. Je suis intervenu auprès de la PV surtout pour dire: "Ça ne sert à rien de faire des descentes comme ça chez les producteurs, ça va mal se passer" et côté producteur je leur expliquais que s'ils voulaient avoir des aides de l'État, du fait de cette calamité, ils avaient tout intérêt à jouer franc-jeu avec la PV. Moi j'étais informé des deux côtés en fait, PV et professionnels. » (responsable de la station INRA).

Pour cela, au cours des nombreux échanges informels qu'ils pouvaient avoir, ses membres ont cherché, à convaincre, d'un coté, les agriculteurs d’être plus transparents sur l'évolution de la situation sanitaire et, d'un autre côté, l'administration de ne pas se montrer trop stricte dans la mise en œuvre des mesures d'éradication.

Nous avons montré comment deux acteurs permettent la mise en place d'arrangements autour des règles relatives à la surveillance sanitaire et facilitent la circulation de l'information entre mondes sociaux. La discussion de la littérature sociologique sur les intermédiaires va nous permettre maintenant d'analyser plus en détail la nature et les motivations de leur action.

\subsection{Des boundary spanners aux «médiateurs de la surveillance»}

Classiquement, la sociologie des organisations a mis en évidence l’importance jouée par certains types d'acteurs dans la réalisation de la collaboration entre organisations. Un certain nombre d'auteurs (Thompson, 1967 ; Aldrich et Herker, 1977) développent les

${ }^{14}$ De fait, le dispositif épidémiologique s’apparente à une expérimentation qui « rend floue la ligne entre le laboratoire et le champ » (Henke, 2000) et au cours de laquelle les scientifiques doivent, pour pouvoir réaliser leur expérimentation, sortir de leur rôle de chercheur de laboratoire pour endosser celui de technicien. 
concepts de boundary role ou de boundary spanner. Ils utilisent ces concepts pour décrire des personnes ou services qui, au sein d'une organisation, ont l'importante fonction de canaliser les échanges d'information et de servir de médiateurs entre l'organisation et son environnement ${ }^{15}$. Prolongeant la réflexion sur ces types d'acteurs, mais critiquant la conception objectifiante de l'environnement sur laquelle elle repose, M. Crozier et E. Friedberg ont - en s'inspirant du travail de H. Jamous ${ }^{16}$ — développé le concept de " marginal-sécant » pour décrire les personnes qui «par leurs appartenances multiples » et " leur capital de relation » mettent en relation les uns avec les autres plusieurs systèmes d'action et jouent ainsi « le rôle indispensable d'intermédiaire et d'interprète entre des logiques d'actions différentes, voire contradictoires » (Crozier et Friedberg, 1977, p. 86). Dans cette perspective, le "marginal-sécant " est considéré comme un acteur stratégique qui tire profit de sa position pour obtenir des gains personnels et pour renforcer sa position au sein de son organisation d'appartenance ${ }^{17}$.

Cette approche, quoiqu'indéniablement heuristique, a deux limites. Premièrement, construite à partir d'une analyse d'interdépendances relativement stabilisées entre des systèmes d'action, elle tend à orienter l'analyse sur les actions routinières sinon récurrentes qui constituent les stratégies des intermédiaires, au risque de ne pas rendre compte de leur nature dynamique et fragile dans les situations moins stabilisées. Or, la situation que nous étudions est une situation relativement exceptionnelle - qualifiée parfois « de crise » par les acteurs - , et relativement instable, évoluant à mesure de la dynamique du virus et des négociations autour de sa prise en charge.

Deuxièmement, reposant sur l'identification par le sociologue des intérêts qui poussent ces acteurs à agir, la sociologie de l'action organisée ne rend pas compte pleinement du sens qu'ils donnent à leur action. Elle ne permet ainsi pas de comprendre pourquoi certains acteurs agissent pour mettre en relation des mondes sociaux alors que leur intérêt à le faire ne paraît pas évident. Dans la situation étudiée, il est possible d'identifier des intérêts pour ces acteurs d'agir comme médiateurs. En effet, par son action, l'agent de la PV obtient de l'information sur l'évolution de la situation sanitaire. Cette information lui est précieuse car il doit mener des prospections sanitaires dans des serres fermées, avec des moyens matériels et humains limités, chez des producteurs qui sont réticents à participer à une politique d'éradication. Cependant, son action l'expose également à être perçu — c'est le cas — par sa hiérarchie comme un agent qui ne mène pas à bien la mission qui lui est donnée de faire appliquer les plans de lutte et de surveillance officiels. Aussi, il pourrait être tout aussi intéressant pour lui d’adopter une stratégie de repli sur le respect strict du droit dans le cadre des moyens limités dont il dispose. La station expérimentale a également des intérêts évidents à agir comme un intermédiaire. Ainsi, elle promeut une plus grande transparence des producteurs qui est pensée par elle comme la condition du maintien de la possibilité future de produire des tomates et, plus spécifiquement pour la station, de continuer à pouvoir mener des expérimentations agronomiques sur cette plante. Par ailleurs, elle met en place une collaboration intéressante d'un point de vue scientifique et financier avec l'équipe de recherche Fractale. Cependant, la station s'expose également à se voir rappeler à l'ordre pour ne pas respecter l'obligation réglementaire d'information et pour s'investir dans des activités qui ne relèvent pas des

\footnotetext{
${ }^{15}$ Dans cette littérature, le raisonnement est fonctionnaliste : il y a nécessité dans une organisation que des personnes soient chargées de relations avec l'environnement, que cela soit explicitement spécifié ou non, que ces activités soient routinières ou non.

${ }^{16} \mathrm{H}$. Jamous étudie la réforme des études médicales et des structures hospitalières. Il insiste, dans ce processus, sur le rôle de marginaux et défavorisés du système médical, en montrant notamment comment leurs représentants poussent la réforme par leur accès à plusieurs lieux de pouvoir et de décision (Jamous, 1969).

17 Pour eux, il est capital de considérer l'environnement comme constitué par l'action des membres de l'organisation, et dans lequel ils jouent à la fois le rôle de " porte-parole des » et de "représentants auprès des » segments concrets de l'environnement (Friedberg, 1993).
} 
missions d'un institut de recherche comme l'INRA mais des autorités sanitaires ou des organisations professionnelles. Nous voyons donc que l'intérêt des deux pivots de la surveillance à favoriser la coopération entre les mondes sociaux n'est pas sans ambiguïté au regard extérieur du sociologue qui voudrait l'analyser.

La sociologie de la traduction (Callon, 1986 ; Latour, 1989) et le concept de «médiateur» qu'elle a développé pour qualifier ceux qui articulent entre eux les différents mondes sociaux permettent de dépasser ces deux limites de l'approche organisationnelle $^{18}$ Cette approche invite en effet à rendre compte de la fragilité des réseaux dans lesquels les acteurs agissent et adopte ce que nous pourrions appeler une perspective pragmatique sur les raisons d'agir des acteurs ${ }^{19}$. Nous allons voir qu'elle permet ainsi de rendre compte de la nature instable et dynamique du travail de liaison réalisé par l'agent de la PV et la station expérimentale et de mieux comprendre les raisons qui poussent ces acteurs à agir comme des médiateurs, au risque même de fragiliser leur position dans leur organisation.

\subsection{Le travail de médiation : transgresser pour surveiller}

L’agent de la PV et la station expérimentale ne sont pas, pour reprendre la distinction faite par la sociologie de la traduction, de simples intermédiaires mais des médiateurs. De fait, ils sont des opérateurs de transformations, de traductions et de distorsions des informations qu'ils peuvent recevoir, dont « les inputs ne permettent jamais de prévoir les outputs » (Latour, 2006). Ils agissent dans les réseaux instables et non formalisés de la « surveillance au concret ». Le directeur de la station INRA ne communique pas immédiatement à l'administration toutes les informations qu'il collecte mais évalue à chaque échange ce qu'il peut ou ne peut pas dire, et à quel moment le dire. Par exemple, ce n'est qu'à l'automne qu'il informe l'agent local de la PV des réunions professionnelles qui ont eu lieu à l'été 2003. L'attitude « accommodante » de cet agent local de la PV l'encourage évidemment à être plus franchement un relais entre le monde professionnel et les autorités sanitaires. De la même manière, le lien que construit l'agent de la PV avec les acteurs professionnels est un lien fragile et dynamique. Non formalisé dans un accord écrit, il repose sur la bonne volonté d'individus à l'entretenir et si l'agent de la PV est invité jusqu'au printemps 2003 à certaines réunions de la cellule de veille, il n’y est plus invité lorsque la situation sanitaire se détériore trop. Servant jusque-là à la fois de source d'aide technique et de relais auprès de l'administration centrale pour demander des aides et un allégement du plan d'éradication, il perd le contact avec les représentants professionnels quand ceux-ci estiment que la priorité est de faire le secret sur le développement du TYLCV et d'empêcher l'administration d'arracher les cultures au moment du cycle de production où elles sont le plus rentables. Cependant, la fragilité de ce lien et sa non-formalisation est en même temps la condition de son existence : c'est parce que les acteurs entrent dans des relations interpersonnelles et ne se lient pas les mains par rapport aux conditions de leurs échanges dans un contexte qui est marqué par une incertitude sanitaire forte qu'ils peuvent, à certains moments, coopérer.

\footnotetext{
${ }^{18}$ Dans cette section, nous ne discutons pas de l'analyse des réseaux sociaux, une autre approche s'étant très sérieusement intéressée à décrire et analyser le rôle des acteurs (brokers) qui sont à la frontière de systèmes d'action - par exemple Gould et Fernandez (1989). Notre terrain d'enquête, marqué par des jeux importants autour de la rétention d'information, ne nous a pas permis d'utiliser les outils qu'elle propose. Plusieurs acteurs ayant refusé d'identifier nommément des personnes avec lesquelles elles étaient en relation, cela rendait difficile la reconstitution des réseaux de circulation d'information.

${ }^{19}$ Ce qui est considéré comme motivant les acteurs, c'est ce qu'ils indiquent comme les motivant en situation. Cette perspective a également orienté des travaux sur l'intermédiation dans d'autres domaines, comme la sociologie de l'art (Hennion, 1983, 1993). Notons que dans une perspective proche, des travaux de sociologie des sciences ont mis en avant les supports matériels sur lesquels reposent les collaborations entre mondes sociaux (Star et Griesemer, 1989 ; Fujimura, 1992).
} 
S’il est possible d'identifier des intérêts orientant le travail de médiation des deux pivots de la surveillance, leur action ne prend pas uniquement sens par rapport à un intérêt personnel. Les entretiens effectués et les observations réalisées soulignent également qu'ils agissent comme ils le font parce qu’ils ont développé, au cours du temps, une capacité d'empathie et ce que D. Chilshom a appelé des «attitudes cosmopolitaines, [...] favorables à l'idée de la coordination entre organisations multiples » (Chisholm, 1989, p. 89). La citation suivante, qui montre l'agent de l'administration se projetant « à la place du producteur » et s'estimant devoir prendre en compte l'intérêt de tous les acteurs concernés, illustre cela :

«Moi je dis, c'est mon point de vue, je suis dans le bateau du service public, de la collectivité, de tout le monde de la profession mais aussi des contribuables, il faut que ce soit juste. Je trouve que sur la base de mon expérience je pense que ce virus est installé durablement, ça sur la base de ce qui s'est passé chez les marocains et les espagnols. On a pu bénéficier de leur expérience mais des virus comme ça, ça ne s'arrête pas comme ça... à partir de là, ce seuil de un pour mille devient un peu dépassé car c'est condamner d'entrée un producteur, on notifie l'arrachage de sa culture mais au bout du compte il n'est pas indemnisé. » (responsable antenne locale PV).

Cette capacité d'empathie se définit comme une capacité à se montrer humain, compréhensif et à prendre en compte les intérêts d'autres acteurs dans la poursuite de son intérêt et la réalisation de son rôle institutionnel (Dupuy et Thoenig, 1985; Thoenig, 1994). Elle est créatrice de tensions pour les médiateurs qui, voulant amener les différents acteurs à coopérer "sans devenir leur otage » (responsable antenne locale PV), redéfinissent au cours de leurs interactions les frontières de ce rôle et l'identité qu'y s'y rattache.

Dans la situation étudiée, trois éléments essentiels participent à la construction de la capacité d'empathie des «médiateurs de la surveillance » envers les producteurs et les techniciens : la circulation dans les espaces professionnels, le partage d'une compétence technique et le temps. En effet, l'un comme l'autre sont présents depuis plusieurs années dans la zone de production. Ils ont participé à de multiples réseaux sociotechniques plus ou moins formalisés dans lesquels ils ont échangé avec des acteurs hétérogènes, producteurs et techniciens, dont ils ont appris à connaître et à comprendre les enjeux, les ressources et les contraintes ${ }^{20}$.

La découverte du virus vient perturber ces réseaux sociotechniques, les relations et les accords sur la valeur de certains biens qui s’y sont développé, sans les faire disparaître. Ayant développé des relations de confiance avec certains producteurs et techniciens, capables de faire preuve d'empathie par rapport à leur situation et valorisant certains enjeux comme le maintien de la production agricole et la coopération entre différents mondes sociaux ${ }^{21}$ les " médiateurs de la surveillance » acceptent, dans une certaine mesure et non sans tension, d'ajuster les règles formelles de la surveillance sanitaire pour travailler à une solution de dépassement des blocages qu'elles induisent.

\section{Remarques conclusives : la surveillance sanitaire comme enjeu politique}

\footnotetext{
${ }^{20}$ Certains ingénieurs de la station expérimentale ont même, au début de leur carrière, travaillé directement en tant que techniciens d'appui aux producteurs. On retrouve là des éléments mis en évidence par D. Chilshom pour caractériser les propriétés d'un système qui favorisent les échanges informels : existence de lieux formels permettant la rencontre des individus ; mobilité des individus entre les organisations qui apportent des relations et des manières de travailler (Chisholm, 1989, p. 122-124).

${ }^{21}$ Soulignons que l'analyse stratégique n’implique pas de ne pas considérer de tels accords sur des valeurs ou des sentiments - cf. la mise au point au chap. 7 de Friedberg (1993). Opposée à la mobilisation de valeurs exogènes dans la compréhension du social, elle laisse une place à la " normativité ordinaire endogène » (Reynaud et Richebé, 2007). Cependant, elle y fait peu de place dans sa définition du « marginal-sécant ».
} 
L'histoire de la surveillance sanitaire révèle, dans tous les domaines, une tension récurrente entre les systèmes reposant sur des principes de confidentialité et de participation volontaire et ceux reposant sur l'obligation et la coercition (Woods, 2004). Du point de vue des pouvoirs publics, la prise en compte de l'intérêt collectif suppose de savoir ce qui se passe dans les espaces privés que sont les espaces de production de végétaux ou les espaces de production de connaissance. Ici, la justification du besoin de dispositifs transparents et obligatoires trouve sa légitimité dans la capacité à circuler des pathogènes, qui rend nécessaire la collectivisation de la lutte contre eux. De par cette capacité à circuler entre différents espaces de production, les pathogènes créent une « solidarité involontaire, vitale pourrait-on dire ${ }^{22}$ entre les différents producteurs, dans notre cas entre les différents propriétaires de plantes. Cependant, il ne suffit pas d'invoquer la coopération et la transparence pour qu'elles se réalisent et notre exemple montre les limites — déjà notées dans d'autres cas du domaine de la santé humaine et animale — d'un dispositif de surveillance formel et coercitif.

Comme nous l'avons montré, la surveillance "au concret " se déploie dans des réseaux d'acteurs qui ont quatre propriétés principales. Premièrement ce sont des réseaux hybrides qui mettent en relation des acteurs de différents mondes sociaux aux intérêts différents et dans lesquels distinguer ce qui relève du technique, du politique ou du scientifique n'est pas toujours aisé ${ }^{33}$. Deuxièmement ce sont des réseaux qui se composent à partir de réseaux déjà existants, antérieurs à l'arrivée de l'objet surveillé. Ils fonctionnaient, par exemple, dans notre cas en vue d'autres finalités que la surveillance du virus. Troisièmement, ce sont des réseaux informels, non coercitifs et autorisant le maintien d'un certain niveau de secret dans les échanges. Dans ces réseaux, les conventions qui organisent les relations entre les acteurs et la diffusion des savoirs produits au cours de la surveillance peuvent être redéfinies à mesure de l'évolution de la situation (l'évolution sanitaire, l'avancée des négociations entre les acteurs) et sa définition par les acteurs. Enfin, nous avons insisté sur le fait que le fonctionnement de ces réseaux reposait sur certains acteurs qui facilitaient la circulation de l'information et la coopération entre mondes sociaux, que nous avons qualifiés de «médiateurs de la surveillance ». Ainsi, nous avons souligné la fragilité de leur action d'intermédiation et montré qu'elle ne traduit pas uniquement la recherche d'un intérêt propre mais est également l'expression d'une capacité d'empathie acquise dans un ancrage local durable.

Ces résultats invitent à développer des travaux — rarement entrepris dans le domaine sanitaire et environnemental (Granjou, 2004 ; Bonnaud, 2005) — qui, au-delà de la dimension institutionnelle et matérielle des dispositifs de surveillance (Demortain, 2006 ; Lezaun, 2006) se donnent les moyens de la compréhension de leur fonctionnement concret. Ces dispositifs visant à recueillir des données sanitaires ou environnementales se sont développés ces dernières années, s’inscrivant dans un mouvement plus général de délégation des pouvoirs publics à des acteurs tiers du contrôle des risques sanitaires ${ }^{24}$ (Borraz, 2004). Notre étude illustre la fragilité et la complexité des conditions de recueil et de circulation des données sur lesquelles les dispositifs de surveillance reposent. Elle montre

\footnotetext{
${ }^{22}$ Nous reprenons ici les termes d'un responsable du service de la PV qui, en 1964, dans la revue professionnelle Phytoma (no 162), défend la nécessité de mettre en place des organisations professionnelles de lutte contre les maladies des plantes.

${ }^{23}$ On renvoie ici à des catégories qui sont souvent utilisées par les acteurs pour indiquer la frontière de leurs activités. La sociologie des sciences et des controverses a bien montré depuis 20 ans comment ces frontières sont poreuses et l'objet d'un travail continu des acteurs.

${ }^{24}$ En France, un décret récent en attente d'application (novembre 2006) relatif à l'épidémiologie dans le domaine de la santé sanitaire animale et végétale vise à encadrer les activités de surveillance en mettant en place des réseaux de surveillance qui seraient alimentés par le travail d’autocontrôle et de traçabilité (Torny, 1998) d'organisations sanitaires professionnelles auxquels les agriculteurs seraient obligés d'adhérer.
} 
qu'on ne peut invoquer la mise en place de dispositifs de surveillance sans prendre en compte la nature politique - au sens de constituant un enjeu dans des réseaux d'acteurs marqués par des relations de pouvoir — des données qu'il vise à recueillir ${ }^{25}$, et sans prendre en compte les traductions multiples (rétention, anonymisation, confinement, etc.) dont ces données font l'objet. Considérer ces éléments comme des propriétés plutôt que comme des biais des activités de surveillance (Barbier, 2006) est un détour indispensable — dans le champ de la sécurité sanitaire et environnementale comme dans d'autres champs — à la compréhension de la «nouvelle politique de la surveillance et de la visibilité » (Haggerty et Ericson, 2006).

\section{Remerciements}

Je remercie les nombreuses personnes qui, par leurs relectures et commentaires, m’ont aidé dans la rédaction de cet article. Merci tout particulièrement : à Marc Barbier de l'INRA ; à mes nombreux interlocuteurs du CSO et notamment à Sophie Dubuisson-Quellier, Pauline Ravinet, Christine Musselin, Olivier Borraz et Julien Barrier; aux participants des " Journées d'étude risques » organisées par TSV (INRA) ; enfin aux relecteurs anonymes du comité de lecture de Sociologie du Travail.

\section{Références}

Aldrich, H., Herker, D., 1977. Boundary Spanning Roles and Organization Structure. Academy of Management Review 2, 217-230.

Barbier, M., 2006. Surveiller pour abattre. La mise en dispositif de la surveillance épidémiologique et de la police sanitaire de l’ESB. Terrains et travaux 11, 101-121.

Bonnaud, L., 2005. Au nom de la loi et de la technique. L’évolution de la figure de l’inspecteur des installations classées depuis les années 1970. Politix 69, 131-161.

Borraz, O., 2004. Les normes : instruments dépolitisés de l'action publique. In: Lascoumes, P., Le Gallès, P. (Eds.), Gouverner par les instruments. Presses de Sciences Po, Paris.

Buton, F., 2006. De l'expertise scientifique à l'intelligence épidémiologique : l'activité de veille sanitaire. Genèses 65 , 71-91.

Callon, M., 1986. Eléments pour une sociologie de la traduction. La domestication des coquilles Saint-Jacques et des marins-pêcheurs dans la baie de Saint-Brieuc. L’Année Sociologique 36, 169-208.

Chisholm, D., 1989. Coordination Without Hierarchy. University of California Press, Berkeley.

Crozier, M., Friedberg, E., 1977. L’acteur et le système. Le Seuil, Paris.

Delos, M., Hervieu, F., Folcher, L., Micoud, A., Eychenne, N., 2005. Dispositif de surveillance biologique du territoire pour le suivi des ravageurs des cultures en France. CIRA, Montpellier.

Demortain, D., 2006. Mettre les risques sous surveillance. L’outillage de la sécurité sanitaire des médicaments et des aliments en Europe. Thèse de l'École normale supérieure de Cachan.

Dodier, N., 1991. Les actes de l’inspection du travail en matière de sécurité : la place du droit dans la justification des relevés d’infraction. In: Chazel, F., Commaille, J. (Eds.),

\footnotetext{
${ }^{25}$ Il s’agit alors de ne pas se limiter à une perspective critique - dominante par exemple dans le champ des surveillance studies, dont on trouvera un état des lieux dans états des lieux dans le numéro spécial de Contemporary Sociology (Marx, 2007) — des formes de disciplinarisation (Staples, 1997) des populations et des sujets qu'implique la mise en place de dispositifs de surveillance.
} 
Normes juridiques et régulation sociale. LGDJ, Paris, pp. 177-188.

Douglas, J.D., 1967. The Social Meanings of Suicide. Princeton University Press, Princeton.

Dufour, B., La Vieille, S., 2000. Epidemiological Surveillance of Infectious Diseases in

France. Veterinary Research 31, 169-185.

Dupuy, F., Thoenig, J.-C., 1985. L’Administration en miettes. Fayard, Paris.

Friedberg, E., 1993. Le pouvoir et la règle. Dynamiques de l'action organisée. Le Seuil, Paris.

Fujimura, J.H., 1992. Crafting Science: Standardized Packages, Boundary Objects, and “Translation”. In: Pickering, A. (Ed.), Science as Practice and Culture. The University of Chicago Press, Chicago, pp. 168-211.

Gould, R.V., Fernandez, R.M., 1989. Structures of Mediation: A Formal Approach to Brokerage in Transaction Networks. Sociological Methodology 19, 89-126.

Granjou, C., 2004. La gestion du risque entre technique et politique. Comités d'experts et dispositifs de trac,abilité à travers les exemples de la vache folle et des OGM. Thèse de l’université Paris-5.

Grmek, M.D., 1993. Le concept de maladie émergente. History and Philosophy of the Life Sciences 15, 281-296. Haggerty, K.D., Ericson, R.V. (Eds.), 2006. The New Politics of Surveillance and Visibility. University of Toronto Press, Toronto.

Henke, C.H., 2000. Making a Place for Science: the Field Trial. Social Studies of Science 30, 483-511.

Hennion, A., 1983. Une sociologie de l'intermédiaire : le cas du directeur artistique de variétés. Sociologie du travail 25, 459-474.

Hennion, A., 1993. La passion musicale : une sociologie de la médiation. Métailié, Paris.

Jamous, H., 1969. Sociologie de la décision. La réforme des études médicales et des structures hospitalières. Éditions CNRS, Paris.

Kollock, P., 1998. Social Dilemmas: The Anatomy of Cooperation. Annual Review of Sociology 24, 183-214. Lascoumes, P., 1993. Construction sociale des risques et contrôle du vivant. Prévenir 24, 23-36.

Latour, B., 1989. La science en action. La Découverte, Paris.

Latour, B., 2006. Changer de société, refaire de la sociologie. La Découverte, Paris.

Lezaun, J., 2006. Creating a New Object of Government: Making Genetically Modified Organisms Traceable. Social Studies of Science 36, 499-531.

Marx, G.T., 2007. Desperately Seeking Surveillance Studies: Players in Search of a Field. Contemporary Sociology 36, 125-130.

Prete, G., Barbier, M., 2004. Du conflit en situation de crise comme révélateur des transformations d'un système professionnel. In: Communication aux journées d'étude « Les conflits d'usage et de voisinage ». INRA, Paris.

Reynaud, J.-D., Richebé, N., 2007. Règles, conventions et valeurs. Plaidoyer pour la normativité ordinaire. Revue franc, aise de sociologie 48, 3-36.

Simmel, G., 1999. Sociologie : étude sur les formes de la socialisation. Puf, Paris.

Staples, W.G., 1997. The Culture of Surveillance: Discipline and Social Control in the United-States. St. Martin's Press, New-York.

Star, S.L., Griesemer, J.R., 1989. Institutional Ecology, “Translations” and Boundary Objects: Amateurs and Professionals in Berkeley’s Museum of Vertebrate Zoology, 1907-1939. Social Studies of Science 19, 387-420.

Thoenig, J.-C., 1994. La gestion systémique de la sécurité publique. Revue française de sociologie 35, 357-392.

Thompson, J.D., 1967. Organizations in Action: Social Science Bases of Administrative Theory. McGraw-Hill, New-York. 
Thurmond, M.C., 2003. Conceptual Foundations for Infectious Disease Surveillance. Journal of Veterinary Diagnostic Investigation 15, 501-514.

Torny, D., 1998. La trac, abilité comme technique de gouvernement des hommes et des choses. Politix 44, 55-71.

Woods, A., 2004. A Manufactured Plague: The History of Foot and Mouth Disease in Britain. Earthscan, Londres. 